\title{
A Simple Reconstruction of GPSG
}

\author{
Stuart M. Shieber \\ Artificial Intelligence Center \\ SRI International \\ and \\ Center for the Study of Language and Information \\ Stanford University
}

\begin{abstract}
Like most linguistic theories, the theory of generalized phrase structure grammar (GPSG) has described language axiomatically, that is, as a set of universal and language-specific constraints on the well-formedness of linguistic elements of some sort. The coverage and detailed analysis of English grammar in the ambitious recent volume by Gazdar, Klein, Pullum, and Sag entitled Generalized Phrase Structure Grammar [2] are impressive, in part because of the complexity of the axiomatic system developed by the authors. In this paper, we examine the possibility that simpler descriptions of the same theory can be achieved through a slightly different, albeit still axiomatic, method. Rather than characterize the well-formed trees directly, we progress in two stages by procedurally characterizing the well-formedness axioms themselves, which in turn characterize the trees.
\end{abstract}

\section{$1 \quad$ Introduction ${ }^{1}$}

Like most linguistic theories, the theory of generalized phrase structure grammar (GPSG) has described language axiomatically, that is, as a set of universal and languagesspecific constraints on the well-formedness of linguistic elements of some sort. In the case of GPSG, these elements are trees whose nodes are themselves structured entities from a domain of eategories (a type of feature structute [6]). The proposed axioms have become quite complex, culminating in the ambitious recent volume by Gazdar, Klein, Pullum, and Sag entitled Generalized Phrase Structure Crammar [2]. 'The coverage and detailed analysis of English grammar in this work are impressive, in part because of the complexity of the axiomatic system developed by the authors.

In this paper, we examine the possibility that simpler deacriptions of the same theory can be achieved through a slightly different, albeit still axiomatic, method. Rather than characterize the well-formed trees driectly, we progress in two stages by procedurally characterizing the well-formedness axioms themselves, which in turn characterize the trees. In particular, we give a procedure which converts GPSG grammars into grammars written

\footnotetext{
${ }^{1}$ This research was made possible by gift from the Systom Dovelopment
} Foundation.

I am indebted to Lauri Karttunen and Ray Perrault for their comments on earlier drafte, and to Roger Fvans, Gurald Gazdar, Ivan Bas, Henry Thompson, and members of the Foundations of Grammar project at the Center for the Study of Language and Information for their helpful discussions during the development of this work. in a unification-based formalism, the PATR-II formalism developed at SRI International (henceforth PATR) [5], which has its own declarative semantics, and which can therefore be viewed as an axiomatization of string well-formedness constraints. ${ }^{2}$

The characterization of GPSG thus obtained is simpler and better defined than the version described by Gazdar et al. 'The semantics of the formalism is given directly through the reduction to PATR. Also, the PA'TR axiomatization has a clear constructive interpretation, unlike that used in Gaydar et al., thus making the system more amenable to computational implementation. Finally, the characteristics of the compilation-the difficulty or ease with which the various devices can be encoded in PATR-can provide a measure of the expressiveness and indispensability of these devices in GPSG.

\section{The GPSG Axioms}

\subsection{A Summary of the Principles}

GPSG describes natural languages in terms of various types of constraints on local sets of norles in trees. Pertinent to the ensuing discussion are the following:

- ID (immediate dominance) rules, which state constraints of immediate dominance anong categories;

- metarules, which state generalizations concerning classes of ID rules;

- LP (linear precedence) rules, which constrain the linear order of sibling categories;

- feature cooccurrence restrictions (FCR), which constrain the feature struetures as to which are permissible categories;

- leature specification defaults (FSD), which provide values for features that are otherwise unspecificd;

and, most importantly,

\footnotetext{
${ }^{2}$ However, cavest is in order that the detailed analysis from this perspec tive of the full range of GPSG devices (especially inmediate dominance (ID) rules, and feature cooccurrence restrictions) is not discussed fully hero, nor do I completely understand them. (See Section 3.4.) And while in a confessional mood, I should add that the algorithm given here has not actually been implemented.
} 
- universal feature instantiation principles, which constrain the allowable local sets of nodes in trees; these feature instantiation principles include the head feature convention (HFC), the foot feature principle (FFP), and the control agreement principle (CAP).

In GPSG all of these constraints are applied simultaneously. $A$ local set of nodes in a tree is admissible under the constraints if and only if there is some base or derived ID rule (which we will call the licensing rule) for which the parent node's category is an extension of the left-hand-side category in the rule, and the children are respective extensions of right-hand-side categories in the rule, and, in addition, the set of nodes simultaneously satisfies all of the separate feature instantiation principles, ordering constraints, etc. By extension, we mean that the constituent has all the feature values of the corresponding category in the licensing rule, and possibly some additional feature values. The former type of values are called inherited, the latter instantiated.

The feature instantiation principles are typically of the follow. ing form: if a certain feature configuration holds of a local set of nodes, then some other configuration must also be present. For instance, the antecedent of the control agreement principle is stated in terms of the existence of a controller and controllee which notions are themselves defined in terms of feature configurations. The consequent concerns identity of agreement features.

\subsection{Interaction of Principles}

Much care is taken in the definitions of the feature instantiation principles (and their ancillary notions such as controller, controllec, free features, privileged features, etc.) to control the complex interaction of the various constraints. For instance, the FFP admits local sets of nodes with slash feature values on parent and child where no such values occur in the licensing ID rule, i.e., it allows instantiation of slash features. But the CAP's abovementioned definition of control is sensitive to the value of the slash feature associated with the various constituents. A simple definition of the CAP would ignore the source of the slash value, whether inherited, instantiated by the FFP, or instantiated in some other manner. However, the appropriate definition of control needed for the CAP must ignore instantiated slash features, but not inherited ones. Say Gazdar et al.:

We must modify the definition of control in such a way that it ignores perturbations of semantic type occasioned by the presence of instantiated FOO'T features. [2, p. 87]

Thus, the CAP is in some sense blind to the work of the FFP. $\Lambda_{s}$ Gazdar et al. note, this requirement makes stating the CAP a much more complex task.

The increased complexity of the principles resulting from this need for tracking the origins of feature values is evident not only in the CAP, but in the other principles as well. The head feature convention requires identity of the head features of parent and linad child. The features agr and slash-features that can be inherited from an ID rule or instantiated by the CAP or FFP, respectively - are head features and therefore potentially subject to this identity condition. However, great care is taken to remove such instantiated head features from obligatory manipulation by the HFC. This is accomplished by limiting the scope of the HFC to the so-called free head features.
Intuitively, the free feature specifications on a category [the ones the HFC is to apply to] is the set of feature specifications which can legitimately appear on extensions of that category: feature specifications which conflict with what is already part of the category, either directly, or in virtue of the FCRs, FFP, or CAP, are not free on that category. [2, p. 95]

That is, the FFP and CAP take precedence (intuitively viewed) over the HFC.

Finally, all three principles are seen to take precedence over feature specification defaults in the following quotation.

In general, a feature is exempt from assuming its default specification if it has been assigned a different value in virtue of some ID rule or some principle of feature instantiation. $[2$, p. 100$\}$

Gazdar et al. accomplish this by defining a class of privileged features and excluding such features from the requirement that they take on their default value. Of course, instantiated head features, slash features, and so forth are all considered privileged. However, a modification of these exemptions is necessary in the case of lexical defaults, i.e., default values instantiated on lexical constituents. We will not discuss here the rather idiosyncratic motivation for this distinction, but merely note that lexical constituent defaults are to be insensitive to changes engendered by the $\mathrm{HFC}$, as revealed ir' this excerpt:

However, this simpler formulation is inadequate since it entails that lexical heads will always be exempt from defaults that relate to their HEAD features.... Accordingly, the final clause needs to distinguish lexical categories, which become exempt from a default only if they covary with a sister, and nonlexical categories, which become exempt from a default if they covary (in relevant respects) with any other category in the tree. [2, p. 103]

Thus the interaction of these principles is controlled through complex definitions of the various classes of features they are applicable to. These definitions conspire to engender the following implicit precedence ordering on the principles, principles earlier in the ordering being blind to the instantiations from later principles, which are themselves sensitive to (and exempt from applying to) features instantiated by the earlier principles.

$$
\mathrm{CAP} \succ 4 \mathrm{FFP} \succ \mathrm{FSD}_{l e x} \succ \mathrm{HFC} \succ \mathrm{FSD}_{\text {nonlex }}
$$

Of course, all ID rules, both base and derived arc subject to all these principles; yet metarule application is not contingent on instantiations of the base ID rules. Conversely, LP constraints are sensitive to the full range of instantiated features. The precedence ordering can thus be extended as follows:

${ }^{2}$ Current efforts by at least certain GPSG practitioners are placing the GPSG type of analysis directly in a PATR-like formalism. This formalism, Pollard's head-driven phrase structure grammar (IIPSG) variant of GPSG, uses a run-time algorithm similar to the one described in this paper [4]. Highly suggestive is the fact that the IPSG run-time algorithm also happens to order the principles in substantially the same way.

"We use the symbol $\rightarrow$ to denote one principle "taking precedence over" another. 


$$
\begin{aligned}
\mathrm{META} \succ \mathrm{CAP} & \succ \mathrm{FFP} \succ \mathrm{FSD}_{\text {lex }} \\
& \succ \mathrm{HFC} \succ \mathrm{FSD}_{\text {noniex }} \succ \mathrm{LP}
\end{aligned}
$$

The existence of such an ordering on the priority of axioms is, of course, not a necessary condition for the coherence of such an axionatic theory. Undoubtedly, this inherent ordering was not apparent to the developers of the theory, and may even be the source of some surprise to them. Yet, the fact that this ordering exist: 3 and is strict leads us to a substantial simplification of the system. Instead of applying all the constraints simultaneously, we might do so sequentially, so that the precedence orderingthe blindness of earlier principles in the ordering to the effects of later ones - emerges simply because the later principles have not yet applied.

This solution harkens back to earlier versions of GPSG in which the semantics of the formalism was given in terms of compilation of the various principles and constraints into pure context-free rules. This compilation process can be combinatorially explosive, yielding vast numbers of context-free rules. Indeed, the whole point of the Gl'SG decomposition is to succinctly express generalizations about the possible phrasal combinations of natural languages. However, by carefully choosing a system for stating constraints on local sets of nodes-a formalism more compact in its representation than context-free grammars-we can compile out the various principles and constraints without risking this explosion in practice.

The GPSG principles are stated in terms of identities of features. What we need to avoid the combinatorial problems of pure CF rules is a formalism in which such equalities can be stated directly, without generating all the ground instances that satisfy the equalities. What is needed, in fact, is a unification-based grammar formalism [6]. We will use a variant of PATR [5] as the formalism into which GPSG grammars are compiled. In particular, we assume a version of $\mathrm{P} \mathrm{N}^{\prime} \mathrm{TR}$ that has been extended by the familiar decomposition into an immediate-dominance and linear-precedence component. This will allow us to ignore the LP portion of GPSG for the nonce.

PATR is ideal for two reasons. First, it is the simplest of the unification-based grammar formalisms, possessing only the appa* ratus that is needed for this exercise. Second, a semantics for the formalism has been provided, so that, by displaying this compilation, we implicitly provide a semanties for GPSG grammars as well. In the remainder of the paper, we will assume the reader's familiarity with the rudiments of the PATR formalism.

\section{The Compilation Algorithm}

We postpone for the time being discussion of the metarules, LP constraints, and feature cooccurrence restrictions, concentrating irstead on the central principles of GPSG, those relating to feature instantiation. The following nondeterministic algorithm generates well-formed PATR rules from GPSG ID rules. A GPSG grammar is compiled into the set of PATR rules generated by this algorithm.

\subsection{Preliminaries}

We first observe that a GPSG ID rule is only notationally distinct from an unordered PATR rule. Thus, the first step in the algorithm is trivial. For example, the ID rule

$$
s \rightarrow X^{2}, I[-s u b j]
$$

is written in unordered PATR as

$$
\begin{aligned}
& X_{0} \rightarrow X_{1}, X_{2} \\
& \left\langle\begin{array}{ll}
X_{0} & n\rangle
\end{array}\right\rangle=- \\
& \left\langle X_{0} v\right\rangle=+ \\
& \left\langle\begin{array}{ll}
X_{0} & \text { bar }
\end{array}=2\right. \\
& \left\langle X_{0} \quad s u b j\right\rangle=+ \\
& \left\langle\begin{array}{ll}
X_{1} & b a r
\end{array}=2\right. \\
& \left\langle X_{2} \quad \Delta u b j\right\rangle=-
\end{aligned}
$$

Note that abbreviations (like $S$ for $[-n,+v, b a r 2,+s u b j]$ ) have been made explicit.

In fact, we will make one change in the structure of categories (to simplify our restatement of the HFC) by placing all head features under the single feature head in the corresponding PATR rule. We do not, however, add an analogous feature foot. ${ }^{5}$ Thus the preceding rule becomes

$$
\begin{gathered}
X_{0} \rightarrow X_{1}, X_{2} \\
\left\langle X_{0} \text { head } n\right\rangle=- \\
\left\langle X_{0} \text { head } v\right\rangle=+ \\
\left\langle X_{0} \text { head bar }\right\rangle=2 \\
\left\langle X_{0} \text { head subj }\right\rangle=+ \\
\left\langle X_{1} \text { head bar }\right\rangle=2 \\
\left\langle X_{2} \text { head subj }\right\rangle=-
\end{gathered}
$$

We use an operation $a d d_{c}$ (read "add conservatively") which adds an equation to a PATR rule conservatively, in the sense that the equation is added only if the equations are not thereby rendered unsolvable. If addition would yield unsolvability, then a weaker set of unifications are added (conservatively) instead, one for each feature in the domain of the value being equated. For instance, suppose that the operation $a d d_{c}\left(\left\langle X_{0}\right.\right.$ head $\rangle=\left\langle X_{1}\right.$ hend $\left.\rangle\right)$ is called for, where the domain of the head fealure values (i.e., the various head features) are $a, b$, and $c$. If the equations in the rule already specify that $\left\langle X_{0}\right.$ heal $\left.a\right\rangle \neq\left\langle X_{1}\right.$ head $\left.a\right\rangle$ then this operation would add only the two equations $\left\langle X_{0}\right.$ head $\left.b\right\rangle=$ $\left\langle X_{1}\right.$ head $\left.b\right\rangle$ and $\left\langle X_{0}\right.$ heal $\left.c\right\rangle=\left\langle X_{1}\right.$ head $\left.c\right\rangle$, since the addition of the given equation itself would cause rule failure. Thus the earlier constraint of values for the a feature is given precedence over the constraint to be added.

In the description of the algorithm, a nomempty path $p$ is said to be defined for a feature structure $X$ if and only if $p$ is a unit path $\left\langle\lambda\right.$ and $f \in \operatorname{dom}(X)$ or $p=\left\langle f p^{\prime}\right\rangle$ and $p^{\prime}$ is defined for $X(f)$. Our notion of a feature's being defined for a constitucnt corresponds to the GPSG concepts of being instantiated or of covarying with some other feature.

As in the previous definition, we will be quite lax with respect to our notation for paths, using $\langle\langle a b\rangle c\rangle$ and $\langle a\langle b c\rangle\rangle$ as synonymous with $\langle a b c\rangle$. Also, we will consistently blur the distinction between a set of equations and the feature structure it determines. (See Shieber [7] for details of the mapping that makes this possible.)

\subsection{The Algorithm Itself}

Now our algorithrn for compiling a GPSG grammar into a P'ATR grammar follows:

\footnotetext{
${ }^{5}$ But recall that alah is a head feature and thus would fall under the path (head slash).
} 
For each ID rule of GPSG (basic or derived by metarule) $X_{0} \rightarrow$ $X_{1}, \ldots, X_{n}$ :

CAP If $X_{i}$ controls $X_{j}$ (determined by $T y p e\left(X_{i}\right)$ and $T y p e\left(X_{j}\right)$ ), then $\operatorname{add}_{c}\left(\left\langle X_{i}\right.\right.$ con $\rangle=\left\langle X_{j}\right.$ con $\left.\rangle\right)$ where

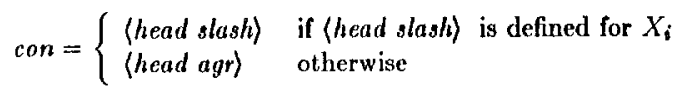

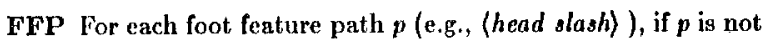
defined for $X_{0}$, then $\operatorname{add}_{b}\left(\left\langle X_{i} p\right\rangle=\left\langle X_{0} p\right\rangle\right)$ for zero or more $i$ such that $0<i \leq n$ and such that $p$ is not defined for $X_{i}{ }^{6}$

FSD $_{\text {lea }}$ For all paths $p$ with a default value, say, $d$, and for all $i$ such that $0<i \leq n$, if $\left\langle X_{i} b a r\right\rangle=0$ and $p$ is not defined for $X_{i}$, then $\operatorname{add}_{c}\left(\left\langle X_{i} f=d\right)\right.$.

HFC For $X_{i}$ the head of $X_{0}, a d d_{c}\left(\left\langle X_{i}\right.\right.$ head $\rangle=\left(X_{0}\right.$ head $)$.

FSD $_{\text {nonlex }}$ For all paths $p$ with a defanlt value, say, $d$, and for all $i$ such that $0<i \leq n$, if $\left\langle X_{i} b a r\right\rangle \neq 0$ and $p$ is not defined for $X_{i}$, then $\operatorname{add} c\left(\left\langle X_{i} \cap=d\right)\right.$.

\subsection{An Example}

Let us apply this algorithm to the preceding rule $R_{1} .^{7}$ We start with the PATR equivalent $R_{\mathbf{s}}$. By checking the existing control relationships in this rule as currently instantiated, we conclude that the subject $X_{1}$ controls the head $X_{2}$. We conservatively add the unification $\left\langle X_{2}\right.$ head agr $\rangle=\left\langle X_{1}\right\rangle$. This can be safely added, and therefore is.

Next, the FFP step in the algorithm can instantiate the rule further. Suppose we choose to instantiate a slash feature on $X_{2}$. Then we add the equation $\left\langle X_{0}\right.$ head slash $\rangle=\left\langle X_{2}\right.$ head slash $\rangle$. Lexical default values recuire no new equations, since no constituents in the rule are given as 0 bar at this point.

The IIFC conservatively adds the equation $\left\langle X_{0}\right.$ head $\rangle=$ $\left(X_{2} \text { head }\right)_{1}$ as $X_{2}$ is the head of $X_{0}$. But this equation, as it stands, would lead to the entire set of equations being unsolvable, since we already have conflicting values for the head feature $s t b j$. Thus the following set of unifications is added instead: ${ }^{8}$

$$
\begin{aligned}
& \left\langle X_{0} \text { head } n\right\rangle=\left\langle X_{2} \text { head } n\right\rangle \\
& \left\langle X_{0} \text { head } v\right\rangle=\left\langle X_{2} \text { head } v\right\rangle \\
& \left\langle X_{0} \text { head bar }\right\rangle=\left\langle X_{2} \text { head bar }\right\rangle \\
& \left\langle X_{0} \text { head agr }\right\rangle=\left(X_{2} \text { head agr }\right\rangle \\
& \left\langle X_{0} \text { head inv }\right\rangle=\left\langle X_{2} \text { head inv }\right\rangle
\end{aligned}
$$$$
\text { .. }
$$

${ }^{6}$ Several comments are pertinent to this portion of the algorithm. First, it is the FFP portion that is responsible for its nondeterminism. Second, the operation add $d_{c}$ is actually superfluous here. The equation can simply be added directly, since we have already guaranteed that the pertinent features are not yet instantiated. By a similar argument, we can conclude that only the $a d d_{c}$ operations in the CAP and HFC are actually necessary. We will use $a d d_{c}$, however, for uniformity. Finally, we assume that an FSD will place the value $\sim$ on any remaining constituents unmarked for foot features.

${ }^{7}$ We do not include here the effect of the rule on every feature postulated by Gazdar et al. but only a representative sample.

${ }^{8} \mathrm{~A}$ more efficient representation of such scts could be achicved by the introduction of nonmonotonic operations such as overwriting or priority union. But such considerations need not concern us here.
Finally, nonlexical defaults are introduced for features not in the domains of constituents. ${ }^{9}$ Since the path (head inv) is defined for the constituents $X_{0}$ and $X_{2},{ }^{10}$ the default value (i.e., '--' according to FSD 1 of Gazdar et al.) is not instantiated on either constituent. Similarly, the case default value (acc, FSD 10) is not instantiated on the subject NP. But the conj feature default $^{11}(' \sim$ ') will be instantiated on all three constituents with the equations

$$
\begin{aligned}
& \left\langle X_{0} \text { conj }\right\rangle=\sim \\
& \left\langle X_{1} \text { conj }\right\rangle=\sim \\
& \left\langle X_{2} \text { conj }\right\rangle=\sim
\end{aligned}
$$

The (partial) generated rule is the following:

$$
\begin{aligned}
& X_{0} \rightarrow X_{1}, X_{2} \\
& \left\langle X_{0} \text { head } n\right\rangle=- \\
& \left\langle X_{0} \text { head } v\right\rangle=+ \\
& \left\langle X_{0} \text { head bar }\right\rangle=2 \\
& \left\langle X_{0} \text { head subj }\right\rangle=+ \\
& \left\langle X_{1} \text { head bar }\right\rangle=2 \\
& \left\langle X_{2} \text { head subj }\right\rangle=- \\
& \left\langle X_{2} \text { head agr }\right\rangle=\left\langle X_{1}\right\rangle \\
& \left\langle X_{0} \text { head slash }\right\rangle=\left\langle X_{2} \text { head slash }\right\rangle \\
& \left\langle X_{0} \text { head } n\right\rangle=\left\langle X_{2} \text { head } n\right\rangle \\
& \left\langle X_{0} \text { head }\right\rangle=\left\langle X_{2} \text { head } v\right\rangle \\
& \left\langle X_{0} \text { head bar }\right\rangle=\left\langle X_{2} \text { head bar }\right\rangle \\
& \left\langle X_{0} \text { head agr }\right\rangle=\left\langle X_{2} \text { head agr }\right\rangle \\
& \left\langle X_{0} \text { head inv }\right\rangle=\left\langle X_{2} \text { head inv }\right\rangle \\
& \cdots \\
& \left\langle X_{0} \text { conj }\right\rangle=\sim \\
& \left\langle X_{1} \text { conj }\right\rangle=\sim \\
& \left\langle X_{2} \text { conj }\right\rangle=\sim \\
& \cdots
\end{aligned}
$$

\subsection{Problems and Extensions}

Several problems have been glossed over in the previous discussion. First, we have not mentioned the role of LP rules. Two possibilities are available for their interpretation: a "run-time" and a "compile-time" interpretation. We can augment the PATR formalism with LP rules in the same way as Gazdar et al., providing for local sets of nodes to satisfy an unordered PATR rule if and only if the nodes are extensions of elements in the ID rule such that the L.P rules are all satisfied. Alternatively, we can generate at compile time all possible orderings of the unordered rules compatible with the LP statements, but this leads us into the problem of interpreting LP statements relative to partially instantiated categories, an issue beyond the scope of this paper.

Second, feature cooccurrence restrictions were ignored in the previous discussion. Again, we will limit ourselves to a brief discussion of the possibilities. One alternative is to modify the lat-

\footnotetext{
${ }^{\circ}$ We have made the simplifying assumption that feature specification defaults are stated in terms of simple default values for features, rather than the more complex boolean conditions used in the Gazdar et al. text. The modifications to allow the more complex FSDs may or may not be straightforward.

${ }^{10}$ The value of the feature head on the constituent $X_{0}$ has the feature inv in its domain because the unification $\left(X_{0}\right.$ head inv $\rangle=\left\langle X_{2}\right.$ head inv $\rangle$ gives as value to $\left\langle X_{0}\right.$ head inv $\rangle$ a variable, the same variable as the value for ( $X_{2}$ head inv). Thus the path (head inv) is defined for $X_{0}$ and, similarly, for $X_{2}$.

"We assume here, contra Gazdar et al., that ' $\sim$ ' is a full-fledged value in its own right, at least as interpreted in this compilation. Since this value fails to unify with any other value, e.g., ' + ' or ' - ', it has exactly the behavior desired, namely, that the feature is prohibited from taking any of its standard values.
} 
tice of categories relative to which unification is defined ${ }^{12}$ in such a way that all categories violating the FCRs are simply removed. Then unification over this revised lattice will be used instead of the simpler version and FCRs will automatically always be obeyed. Unfortunately, the possibility exists that unification over the revised lattice may not bear the same order-independence properties that characterize unification over the freely agenerated lattice. Of course, if this turns out to be the case, it casts doubt on the well-foundedness of the original Gazdar at al. interpretation of FCRs as well, and thus is an interesting question to pursue.

Another alternative involves checking the FCRs at every point in the algorithm, throwing out my rules which violate them at any point. In addition, FCRs would be required to be checked during run-time as well. This alternative, though more direct, violates the spirit of the enferprise of giving a compilation from the complex Gazdar et al. formulation to a simpler system.

$A$ final problen coneerns the ordering of the $11 \mathrm{l} C$ and the CAP. The definitions of controller and controllee necessary for stating the CAP depend on the assignment of semantic types to constituents, which in turn depend on the configuration of fertures in the categories. We have already noted that the features pertinent to the defitition of semantic type (und hence control) do not include instantianted foot features. Indeed, Gazdar et al claim that "it is just HCAD feature specifications (other than those which are also POOT feature specifications) and inlerited NOO' Peature specifications that determine the semantic types relevant to the clefinition of control." $[2,0.87]$ Unfortunately, the ordering we have given precludes instantiated head fentures from participaling in the definition of semiantic type and hence the CAP' is It secms that the IINO must apply before the $O A P^{x}$ for the desinition of semantic type, but after the CAP' so that the CAP instantiations of head features take precedence. 'T'hus, our earlier claim of strict ordering may be falsified by this case.

Of course, the set of fentures necessury for type determination and the aet instantiated by the CAP' may be disjoint. In this case, we can merely split the application of the IIFC in two, instantiating the former elass before the CAP and the luter class after the FWP as originally described. Alternatively, it might be possible to notate head features on the liead constituent rather than the parent as is conventially done. In this ense, the information needed by the CAP is inherited, not instantinted, head feature values, and thus not subjeet to the ordering problem.

On the other hand, if the sets are nondisjoint, this presente a problem not only for our algorithric analysis, but for the definition of GPSG given by Gazdar et al. Suppose that the IIPC determines types in such a way that the $C A P^{3}$ is required to apply and instantiates head features thereby overriding the original values (since the CAP' takes precedence) and changing the type determination so that the CAP does not apply. We would thus icquire the CAP to apply if and only if it does not apply. This paradox appears as an ordering cycle in our algorithm; in the declarative definition of Gazdar ot all, it would be manifested in the inindmissability of all local sets of nodes [1], an expually unattractive effect. We leave the resolution of this problern open for the time being, merely noting that it is a dificulty for GPSC: in general, and not only for our characterization.

${ }^{12}$ For the technical background of such a move, see the discussion of PATR semantics [3].

${ }^{13}$ I am indebted to Roger Lvans and Willian Keller for pointing this problem out to me and for helpful discussion of solution alternatives.

\section{Conclusion}

The axiomatic formulation of generalized phrase structure gramnar by Gazdar et al. is a quite subtle and complex system. Yet, as we have shown, GPSG grammars can be substantially con verted to grammars in a simpler, and constructive, axiomatic system through a straightforward (albeit procedural) mapping. Intrinsic in this conversion is the use of a unification-based grammar formalism, so that axioms can be stated schematically, without enumcrating all of their possible instantiations. In fact, we would contend that defining the semantics of a GPSG grammar in this way yields a much simpler formulation. The need for such a reconstruction is evident to anyone who has studied the Gazdar ct al, texi.

Of course, even if certain parts of the GPSG formalism not discussed fully here, i.e., FCRs and LP' constraints, are found not to be reducible to PATR, this in itself would be an interestingr fact. It would show that exactly those porticns of the formalign were truly essential for stating certain analyses, i.e., that analyses using, those formal devices do so necessarily.

We find a hopeful sign in the recent work in GPSG that is procerding in the direction of using unification directly in the rules, in addition to its implicit use in feature instantiation prineiples. We hope that this paper hats provided evidence that such a system may be able to more simply state the kinds of generalizations that linguists claim, and has pointed out both the possibilities and difficulties inherent in these techniques.

\section{Hererences}

[1] Gerald Gazdar. Personal communication, 1986.

[2] Gorald Gaydar, Lwan KIcin, Gcoffrey K. Pullum, and Ivan A. Sag. Ceneralized Phrase Structure Crrammar. Blackwell Publishing, Oxford, lingland, and Harvard Universily Press, Cambridge, Massachusetts, 1985.

[3] Fenando C. N. l'ercira and Stuart M. Shieber. The seman ties of grammar formalisus seen as computer languages. In Procedings of the Tenth International Conjerence on Com. putational linguistics, Stanford University, Stanford, California, 2.7 July 1984 .

[1] Carl Pollard. Lecture notes on head-driven phrase-structure grammar. Vebruary 1985. Center for the Study of Language and Information, umpublished.

[5] Stuart M. Shieber. The design of a computer language for linguistic information. In Procedings of the Tenth International Conference on Computational linguistics, Stanford University, Stanford, California, 2-7 July 1981

[6] Stuart M. Shieber. An Introduction to Unification-Based Ap. prouches to Grammar. CSLI Lecluse Note Series, Center for the Study of I,anguage and Information, Stanford, California, lortheoming.

[7] Stuart M. Shieber. Using restriction to extend parsing algorithms for complex-feature-based formalisms. In Proceedings of the 22nd Annual Metting of the Association for Computational linguistics, University of Chicago, Chicago, Illinois, July 1985. 